\title{
PHOTOMETRIC VARIABILITY IN EARLY B STARS I.
}

\author{
53 ARIETIS
}

\author{
SALVADOR GONZÁLEZ BEDOLLA \\ Instituto de Astronomía, UNAM, México Apartado Postal 70-264, C.P. 04510, México, \\ D.F. México
}

Known as well as $\beta$ CMa stars, the classical $\beta$ Cep stars are a group of variables of spectral type between $\mathrm{BO}$ to $\mathrm{B} 2$ and luminosity classes II to IV with light and radial velocity periods between 3 and 7 hours and radial velocity ranges usually $<50 \mathrm{~km} / \mathrm{sec}$. Their photometric amplitudes are usually $<$ $0.1 \mathrm{mag}$ in the visible. The light curves are approximately sinusoidal (however, multiple modes are frequently detected with low amplitudes), which lag about $90^{\circ}$ behind the radial velocity curve and the color changes in the blue and visual spectral regions are small. Color and luminosity show that maximum temperature coincides with minimum radius. Probably not all the stars in this spectral range are variables; most investigators searching in this region for $\beta$ Cep variables have observed that a large number of stars in this zone are constant in light. Also, the $\beta$ Cep stars are not different spectroscopically from nonvariable stars of the same MK type.

Recently, however, new $\beta$ Cep stars have been detected and with the new types of variables discovered near this zone, like the faint OB stars (mostly found in associations, Hill 1967), the "53 Per" variables, the "ultrashort" period, the "slow" and Be variables, it is now clear that the classical box of instability for B stars does not have a well delimited border. Hence, with the small number of new groups of variables discovered, one is led to the conclusion that some of the stars in these new "groups" must be monitored in order to know if they are really different from the classical $\beta$ Cep stars.

With the aim of detecting new variables of the types mentioned above, we present here a possible new variable star situated near the zone discussed above; this star, 53 Ari $(\mathrm{B} 2 \mathrm{~V}, \mathrm{~V}=6.1)$ is located near the red edge of the classical instability box where the $\beta$ Cep stars are. The comparison stars were $C_{1}=\mathrm{HR} 948(\mathrm{~B} 8 \mathrm{~V}, \mathrm{~V}=5.9)$ and $C_{2}=\mathrm{HR} 972(\mathrm{~A} 0 \mathrm{IV}-\mathrm{V}, \mathrm{V}=4.9)$; the observations were made on the nights of Oct. 7, 1980 and Oct. 19 and 20, 1982 at the National Astronomical Observatory in San Pedro Mártir, Baja California, México, with the $1.5 \mathrm{~m}$ telescope, the Johnson B filter and a DC photoelectric photometer equipped with 1P21 photomultiplier tube cooled with dry ice; a voltage to frequency converter provided digital read out of the output. The observational sequence followed was, uninterruptedly: $C_{1}$, $\mathrm{V}, C_{2}, \mathrm{~V}, C_{1}, \ldots$ on all nights, each observation consisted at least of five 10 -second integrations of the star followed by one of the sky.

The result of subtracting the mean of the magnitudes of the comparison 
stars from the magnitude of the variable star $(\Delta B)$ are plotted and shown in the figure along with $\left(C_{1}-C_{2}\right)$. The accuracy of each observation is 0.004 mag; time is reported in UT and its accuracy is of 1 minute.

The light curve obtained for 53 Ari shows that on the night of Oct. 7, 1980 the star was probably variable: the light curve shows a clear oscillation with $\mathrm{a} \approx 0.02 \mathrm{mag}$ amplitude and a period between 0.5 and $1 \mathrm{~h}$ with a modulation that indicates that other frequencies are probably present. The dispersion for the difference $C_{1}-C_{2}$ on this night $(0.008 \mathrm{mag})$ is only $40 \%$ of the total amplitude of the light curve. In contrast, on Oct. 19, 1982, the star was constant since the dispersion for $\Delta \mathrm{B}$ and $C_{1}-C_{2}$ is the same $(0.008$ mag) and apparently there is no clear oscillation for the light curve of the star observed. The star was also constant during most of Oct. 20,1982, but, surprisingly, showed a sudden, short and bright little increase (like a flash) of $0.01 \mathrm{mag}$ approximately in a elapsed time of around one hour.

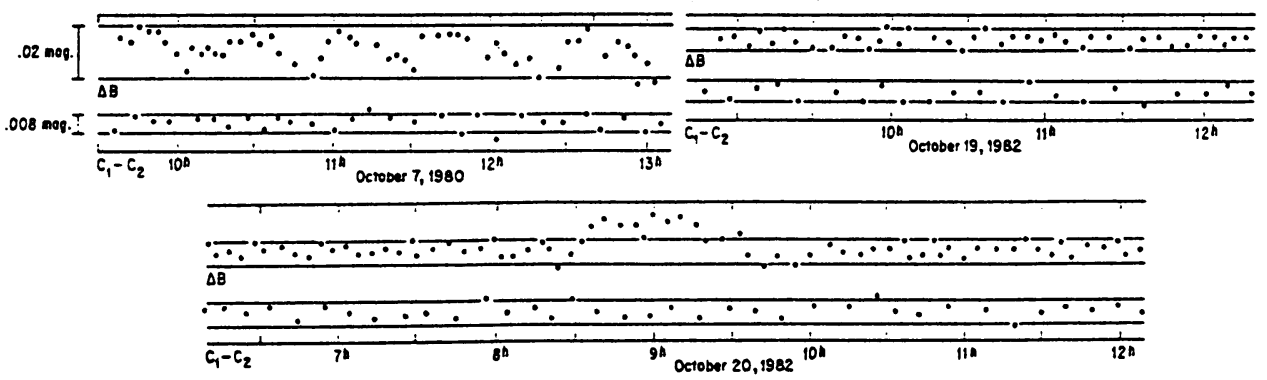

This photometric behavior of 53 Ari resembles in some sense 53 Psc (B2IV, V=5.9) which is located on the red edge of the "instability box" with a controversial photometric behavior where a peculiar amplitude modulation or transient variability may better account for the observations made for this star (Jerzykiewicz and Sterken, 1990) and the observed amplitude variation could be related to the location of $53 \mathrm{Psc}$ at the low temperature border of the $\beta$ Cep instability strip where the mechanism responsible for the instability is perhaps not efficient enough to maintain a stable pulsation (Le Contel et al. 1988). In this case, another possibility is that 53 Psc presents long-term changes in amplitudes similar to those pointed out with different $\beta$ CMa stars (Chapellier, 1986). These suggestions could be applied to 53 Ari too. However, the little short flash observed on Oct. 20, 1982 is not explained with these ideas. In any case, a continuous and careful photometric monitoring for 53 Ari will be very useful in order to confirm the observations presented here. Recently, differential photoelectric Strömgren photometry was made for 53 Ari. The reductions are in progress.

Chapellier, E., 1986, Astron. Astrophys. Suppl. Ser., 64, 275.

Hill, G., 1967, Ap. J. Suppl. 130, 263.

Le Contel et. al., 1988, IBVS No. 3131.

Jerzykiewicz, M. and Sterken C., 1990, Astron. Astrophys. 227, 77. 